using multiplex flow-cytometry. Total RNA was then isolated from the CD8T cells and were subjected to lncRNA and mRNA gene expression analysis.

Results The isolated exosomes were $30-180 \mathrm{~nm}$ in diameter and appeared as cup-shaped vesicles. The exosomes were positive for TSG101, CTLA4 and FAS expression. Interestingly, results from the apoptosis assay indicated that leanCRC exosomes were able to induce a higher percentage of CD8T cell death, in comparison to obeseCRC exosomes $(\mathrm{p}<0.01)$. Similarly, this observation was also seen in the nonCRC exosomes. Furthermore, in the cytokine analysis, leanCRC exosomes induced a higher release of Perforin and IFNG than obeseCRC exosomes $(\mathrm{p}<0.05)$. Notably, this observation was inverted in the nonCRC exosomes. Interestingly, obeseCRC exosomes induced higher levels of sFas and sFasL than leanCRC. Moreover, the microarray analysis showed that most of the dysregulated lncRNAs and genes were involved in the oxidative stress pathways.

Conclusions Contrary to our hypothesis, leanCRC exosomes were more immunosuppressive than obeseCRC exosomes, particularly in relation to CD8T cells. However, effects on other immune markers should be investigated as well.

\section{IDDF2019-ABS-0107 CLINICAL PROFILE AND OUTCOME OF ACUTE PANCREATITIS IN CHILDREN ADMITTED IN PHILIPPINE CHILDREN'S MEDICAL CENTER}

Emaluz Parian*, Marilou Tan, Randy Urtula, Maria Estela Nolasco. Philippine Children's Medical Center, Philippines

\subsection{6/gutjnl-2019-IDDFabstracts.27}

Background Acute pancreatitis, once considered rare in children, has been reported to show an increasing incidence worldwide. Determining Philippine data on clinical profile, factors, severity and outcome would help improve the recognition, diagnosis and management of this emerging disease.

To determine the clinical profile of children with Acute Pancreatitis and the factors associated with their outcome and severity. Methods A retrospective cohort study was done on children 0-18 years old with a diagnosis of Acute Pancreatitis. Demographic, clinical and diagnostic data gathered were compared among severity classification and outcome.

Results Thirty-five cases were identified in a period of 18 years, but only 28 cases were reviewed. Mean age was 11.5 years old \pm 4.1 SD (range 4 - 18) with slight male predominance. Ninety-three percent presented with abdominal pain. Most common etiology was idiopathic (44\%), followed by biliary disease and infection (21\% each). Gallstones and choledochal cyst post excision were the most common of the comorbidities seen. Most common imaging findings was that of an edematous or enlarged pancreas. Five cases of acute recurrent pancreatitis noted. Most common local complications seen were pseudocyst formation and fluid collection with 11\% each. One death was due to hemorrhagic pancreatitis seen intraoperatively. Of the factors reviewed, gallstones and the 3-10 years old age group were found have a statistically significant difference in terms of having a mild vs non-mild severity classification and type of clinical outcome on discharge, respectively.

Conclusions In this study, gallstones and age group of 3-10 years are found to affect severity and outcome and should be considered closely during management of pediatric acute pancreatitis.
A multi-center study on the incidence and clinical profile and outcome is recommended to obtain a better picture of the acute pancreatitis in Filipino children to help clinicians in recognizing and decreasing the morbidity and mortality of this disease.

\section{IDDF2019-ABS-0113 FGF14 IS A FUNCTIONAL TUMOR SUPPRESSOR THROUGH INHIBITING PI3K/ AKT/MTOR PATHWAY IN COLORECTAL CANCER}

Tianhong Su*, Ning Zhang, Linlin Huang, Guangyan Wei, Sui Peng, Zhirong Zeng. The First Affiliated Hospital of Sun Yat-sen University, Sun Yat-sen University, China

\subsection{6/gutjnl-2019-IDDFabstracts.28}

Background Colorectal cancer (CRC) is one of the most common malignancies worldwide. Emerging evidence indicates that promoter methylation of genes associated with gene silencing, plays an important role in the development and progression of CRC. In this study, we aimed to investigate the epigenetic regulation, biological function and molecular mechanism of FGF14 in CRC.

Methods The expression of FGF14 in CRC cell lines, CRC tissues and paired adjacent normal tissues was detected by PCR and Western blot. The biological function of FGF14 in CRC was interrogated by cell viability assay, colony formation, flow cytometry, cell invasion and migration assay, as well as in vivo study.

Results We found FGF14 was downregulated or silenced in all $(10 / 10)$ CRC cell lines, while it was expressed in normal colonic tissues. The expression of FGF14 was lower in primary CRCs as compared to their adjacent normal tissues. Significant higher methylation of FGF14 was observed in CRCs than that in normal tissues based on the data from TCGA database. The loss of FGF14 gene expression was restored by treatment with DNA methyltransferase inhibitor 5-Aza. Reexpression of FGF14 in CRC cell lines inhibited cell viability and colony formation, and induced cell apoptosis. In xenograft mouse model, overexpression of FGF14 significantly reduced tumor growth $(P<0.001)$. FGF14 induced mitochondrial apoptosis and inhibited PI3K/AKT/mTOR pathway.

Conclusions In conclusion, FGF14 is a novel tumor suppressor, which suppresses cell proliferation and induces cell apoptosis via mediating PI3K/AKT/mTOR pathway.

\section{IDDF2019-ABS-0120 PAN-CANCER STUDY OF PROGRESSIVE PROTEIN SIGNATURES FOR DIGESTIVE CANCERS}

${ }^{1}$ Tao Zeng*, ${ }^{2}$ Xiangtian Yu, ${ }^{1}$ Chengming Zhang. ${ }^{1}$ Key Laboratory of Systems Biology, Institute of Biochemistry and Cell Biology, Chinese Academy Science, China; ${ }^{2}$ Shanghai Jiao Tong University Affiliated Sixth People's Hospital, China

\subsection{6/gutjnl-2019-IDDFabstracts.29}

Background As functional executors, protein signatures will be more sensitive than gene signatures in characterizing biological systems. The direct study of the functional proteome is capable to complement and extend omics analysis. Especially, combining cancer research with proteome analysis can identify protein biomarkers to distinguish disease types or subtypes. Methods The progressive signatures during dynamical biological processes are attracting more and more attention. As an 